\title{
Mania in a Patient With Multiple Myeloma After Using Bortezomib
}

\author{
Huinan Jiang ${ }^{\mathrm{a}}$, Rong $\mathrm{Hu}^{\mathrm{a}}$, Miao Miao ${ }^{\mathrm{a}}$, Bin $\mathrm{Wu}^{\mathrm{a}}$, Zhuogang Liu ${ }^{\mathrm{a}}$, b
}

\begin{abstract}
Multiple myeloma (MM) is caused by a clonal proliferation of malignant plasma cells and ranks the second most frequent malignancy of hematology. Despite emerging intensive therapies including new agents and high-dose treatment with stem-cell transplantation, MM still remains an incurable neoplastic disease. Bortezomib was the first proteasome inhibitor (PI) which can improve response rates and overall survival in both newly diagnosed and recurrent MM patients with few adverse effects. The patient was a 61-year-old woman with MM who suffered from mania after initially receiving chemotherapy regimen of PTD (bortezomib, thalidomide, dexamethasone). The regimen was forced to stop due to the severe adverse effect. Then the symptoms were relieved and the patient was given VAD (vincristine, adriamycin, dexamethasone) regimen, and mania was never performed again. It has not been shown that bortezomib can lead to mania in MM patients in the introduction of the drug. Timely detection, immediately stopping related drugs and treating the primary disease actively are key steps for improvement. Poor psychosocial state is another crucial factor which ought to be taken into account in the prevention and treatment of mania occurrence.
\end{abstract}

Keywords: Mania; Multiple myeloma; Bortezomib; Drug-related side effects and adverse reactions

\section{Introduction}

Bortezomib is a boronate-based dipeptidyl proteasome inhibitor (PI) that primarily targets the chymotrypsin-like activities of the intracellular proteasome enzyme complex, and has been approved by the US Food and Drug Administration in 2003

Manuscript accepted for publication October 22, 2014

aHematology Department, Shengjing Hospital, China Medical University, Shenyang, China

${ }^{\mathrm{b} C}$ Corresponding Author: Zhuogang Liu, Hematology Department, Shengjing Hospital, China Medical University, Shenyang 110020, China.

Email: sj_liuzg@163.com

doi: http://dx.doi.org/10.14740/jmc1979w based on large multicenter trials [1]. According to the existing studies, mania might be associated with dopamine $\mathrm{D} 2$ receptor, GSK-3 (glycogen synthase kinase), protein kinase C, inositol monophosphatase overactivity, increased arachidonic acid turnover or cytokine synthesis. Here we report a patient with MM who suffered from mania after receiving chemotherapy regimen of PTD (bortezomib, thalidomide, dexamethasone). According to our knowledge, there have been few reports of mania as an adverse effect of bortezomib.

\section{Case Report}

The patient was a 61-year-old woman who complained with "feeling tired for 2 months and vomiting in the recent 10 days" and had a rib fracture due to trauma a month ago with no other diseases in the past. She completed basic examinations as follows: white blood cell (WBC) count, $5.3 \times 10^{9} / \mathrm{L}$; hemoglobin, $58 \mathrm{~g} / \mathrm{L}$; platelet count, $101 \times 10^{9} / \mathrm{L}$; creatine, $322 \mu \mathrm{mol} / \mathrm{L}$ (normal range: 53 - $155 \mu \mathrm{mol} / \mathrm{L}$ ); urea nitrogen, $10.95 \mathrm{mmol} / \mathrm{L}$ (normal range: $2.5-7.2 \mathrm{mmol} / \mathrm{L}$ ). After application with pantoprazole sodium and infusion with red cell suspension, the patient felt no better. Then she completed further examinations as follows: albumin, $32 \mathrm{~g} / \mathrm{L}$ (normal range: $35-53 \mathrm{~g} / \mathrm{L}$ ); uric acid, $798 \mu \mathrm{mol} / \mathrm{L}$ (normal range: 142 - $420 \mu \mathrm{mol} / \mathrm{L}$ ); $\mathrm{Ca}^{2+}, 3.23$ $\mathrm{mmol} / \mathrm{L}$ (normal range: $2.1-2.55 \mathrm{mmol} / \mathrm{L}$ ). In consideration of her hypercalcemia, elevated serum globulin, abnormal renal function, and anemia, we supposed that it might be myeloma or other neoplastic tumors. Therefore, the patient received further more examinations and was carried on bone marrow aspiration. The report showed as multiple myeloma (MM). This profile assessed the diagnosis of stage III according to Durie Salmon standard and she was divided into B group due to poor renal function. Then she received PTD regimen. It was worth mentioning that bortezomib was administered at the dose of $1.3 \mathrm{mg} / \mathrm{m}^{2}$ on days $1,8,15$, and 22 with dexamethasone $15 \mathrm{mg}$ intravenous infusion on the day and the day after bortezomib was used, and thalidomide $75 \mathrm{mg}$ was orally given every night for 35 days. On the ninth day of the chemotherapy, the patient suffered from mental disorder, performing as in high spirits, irritable, restless, balderdash and less sleep; she also sometimes thought that she had great power to solve problems that she was not able to finish under normal circumstances. We reexamined her creatine increasing to $663 \mu \mathrm{mol} / \mathrm{L}$, so she was 


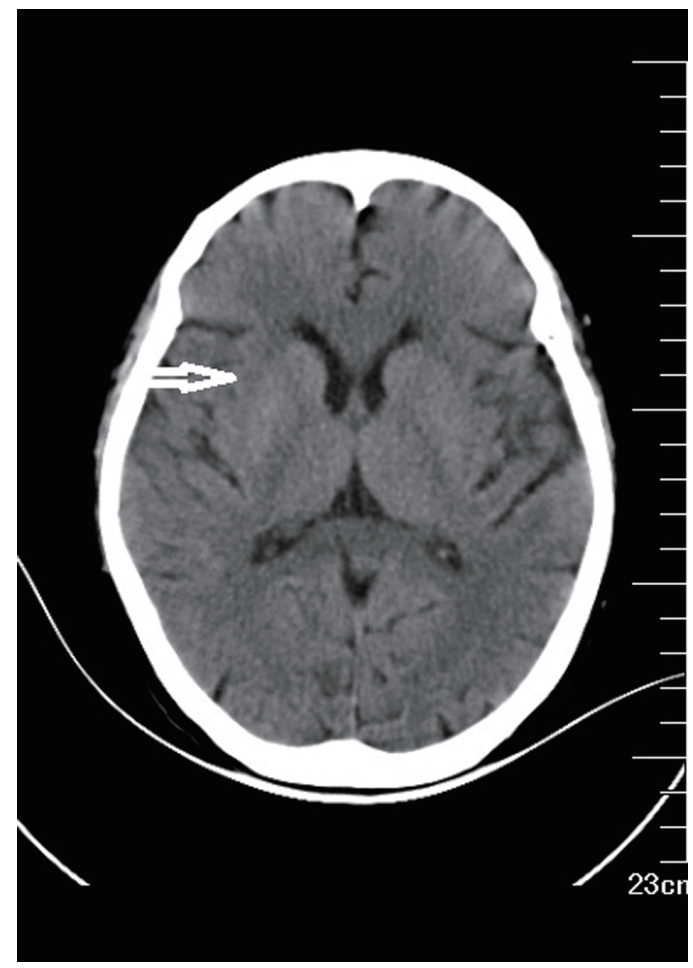

Figure 1. The head CT showed that there existed a hypodense shadow in the right parietal-occipital area adjacent to the lateral ventricle, indicating cerebral infarction possibly.

given hematodialysis three times a week. Only after the first hematodialysis, her psychiatric symptoms improved. From then on, her creatine was fluctuated and the psychiatric symptoms never relieved again after hematolysis. During this time, she received head CT, showing that there existed only a small hypodense shadow in the right parietal-occipital area adjacent to the lateral ventricle, indicating cerebral infarction possibly with no organic lesion (Fig. 1). So it was considered that the psychiatric symptoms were associated with the side effects of certain drug. Therefore, we reduced the dosage of bortezomib to $1.0 \mathrm{mg} / \mathrm{m}^{2}$ and dexamethasone to $10 \mathrm{mg}$, but the dose of thalidomide did not change. During this time, the patient carried on head MRI, and the report showed that there existed punctate lacunar foci or demyelinating lesions under the cortex of right frontal and parietal lobe and leukoaraiosis on lateral forefoot (Fig. 2). However, on the 16th day of the chemotherapy, the patient suffered from the above psychiatric symptoms again, so we stopped bortezomib and dexamethasone, and asked for consultation of psychological department. The psychological doctor combined her psychiatric symptoms, such as always in high spirits, irritable, restless, balderdash, less sleep, hypermetamorphosis and the disease course continued over 1 week, diagnosing the patient as mania and gave her olanzapine 10 mg QD PO and depakine 1.0 g QD PO to improve the central nervous system symptoms for 1 month. Fortunately, the patient finally relieved and at later time received VAD (vincristine, adriamycin, dexamethasone) regimen, and she had never had mental disorder hereafter.

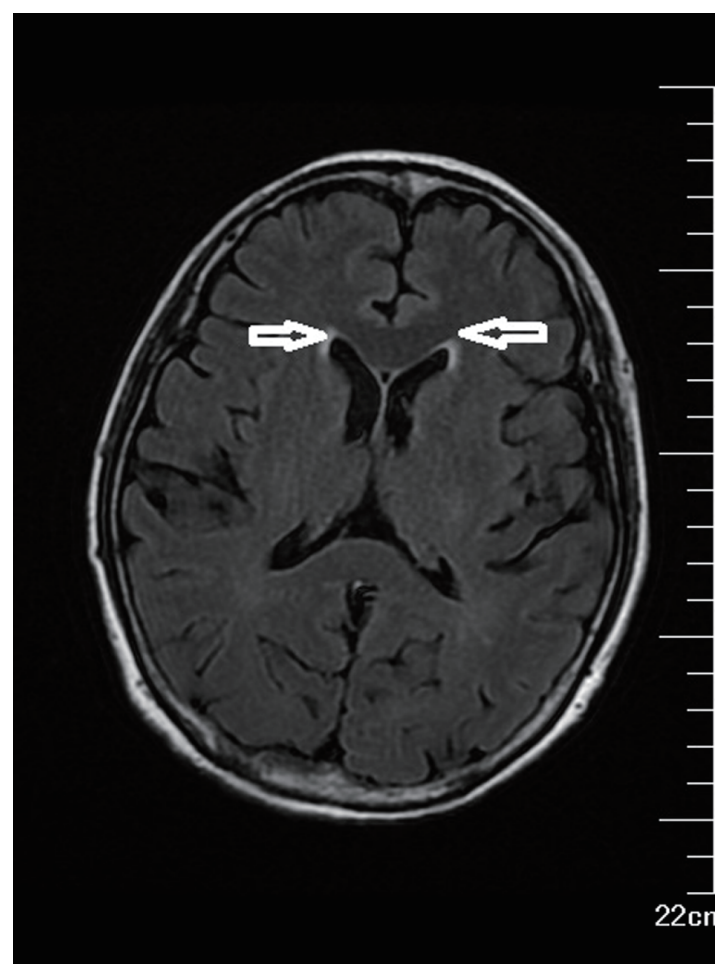

Figure 2. The head MRI showed that there existed punctate lacunar foci or demyelinating lesions under the cortex of right frontal and parietal lobe and leukoaraiosis on lateral forefoot.

\section{Discussion}

MM is a neoplastic disease [2] which will progress to involve many important systems of the body. From the perspective of the disease, psychiatric symptoms may be relative to the following mechanisms: 1) direct infiltration of tumor cells; 2) renal failure; 3) hypercalcemia; 4) hypoxic ischemic encephalopathy. Combined with the incurability of MM, this situation makes myeloma patients an especially vulnerable group. It has been reported that the psychiatric symptoms happened in renal failure always appearing as neurasthenic syndrome, depressive state with taciturn and silence, but our patient was in high spirits and talkative. In addition, according to this case, the patient relieved after the first hematodialysis, but relapsed soon and the following hematodialysis could not improve the symptoms. This proved that her psychiatric symptoms were not in parallel with her renal function, so we thought that the mental disorder was not related to renal failure. Also, it has been reported that hypercalcemia can induce mental disorder. The existed reports illuminate that only when blood calcium exceeds $4.5 \mathrm{mmol} / \mathrm{L}$, it will probably induce psychiatric symptoms. The mechanism is that hypercalcium has toxic effect on cerebral cells and can interfere electrophysiological activities. However, in our case, when she had mental disorder, her blood calcium was in the range of $2.06-2.62 \mathrm{mmol} / \mathrm{L}$, and when she was in the most severe manic state, her blood calcium had already decreased to normal. According to the above, we supposed that the mania 
was not related to hypercalcium. During the whole course, the liver function of the patient was in normal range and she had no jaundice or any typical behavior change like hepatic encephalophathy, so we rule out this possibility. As we have known, cerebral infarction can also cause mental disorder. Most cases happen in patients who have hypertension, cerebral arteriosclerosis even with stroke, or cerebral circulation insufficiency history. The clinical manifestations always in fluctuating process show ecmnesia, irritable, sometimes with delusion. But the patients always have normal cognitive function in a period, can perceive their own abnormal behavior, and then ask for doctors for themselves. At the same time, the patients usually suffer from some neurological signs according to the infarction lesions, such as hemiplegia, nystagmus, agnosia, ataxia, or other pyramidal signs. Cerebral infarction was also excluded because our patient had no cerebrovascular disease in the past and her blood pressure was in normal range throughout the course. She had no cognitive function when she was in mental disorder, and could not perceive her abnormalities in time. Furthermore, her head CT showed that there existed a hypodense shadow in the right parietal-occipital area adjacent to the lateral ventricle, but we could see that the infarction lesion was small which would not cause such psychiatric symptoms in normal conditions. Even in her MRI, we cannot see the typical image of "punched out" destructions in skull as myeloma cells infiltrating brain. Therefore, we analyzed that the mania might be associated with some drug and interrupted the regimen except that she had thalidomide $75 \mathrm{mg}$ QN PO during the whole course. In addition, the patient received VAD regimen afterwards, and did not appear mania again, so it might not be related to the side effects of thalidomide or dexamethasone and support the idea that mania was associated with bortezomib. There have been no reports related to the side effect of bortezomib to induce manic state. The PI bortezomib (Velcade, PS341) can induce apoptosis, reverse drug resistance of MM, and also affect their micro-environment by blocking cytokine circuits, angiogenesis and cell adhesions [3]. It has been reported that bortezomib can cause reversible posterior leucoencephalopathy (RPLS), which is commonly caused by hypertensive encephalophathy, immunosuppressants, or chemotherapy [4]. The clinical manifestations of RPLS include seizures, headache, altered mental state and visual loss, which is to some extent similar to our case. But reports describe highly typical neuroimaging appearances comprising white matter edema affecting the posterior parieto-occipital lobes of the cerebral hemispheres, usually bilaterally. The image of our MRI is quite different from the above descriptions. Therefore, it is excluded that the psychiatric symptoms are caused by RPLS. It has been reported that mania is closely related to dysfunctions of phenylephrine and 5-hydroxytryptamine system, as well as abnormalities of electrolyte metabolism. Up to now, it is still not clear that if bortezomib has something to do with these mechanisms. Bortezomib is used by its function on apoptosis of myeloma cells, but is also mentioned that it can improve cerebral ischemia and hypoxia to protect brain cells survival. It can decrease degradation of $\mathrm{I} \kappa \mathrm{B}$, consequently suppressing the activity of NF- $\mathrm{KB}$, and decreasing the expression of inflammatory factors such as IL-1, TNF and cell adhesions [5]. These can decrease the aggregation of neutrophils in cerebral microvessels and improve the "no-reflow" phenomenon caused by neutrophils blocking in microcirculation system. Therefore, bortezomib has "double-edged" functions dose independently. It has been found that bortezomib weekly used can achieve similar CRs and cause less side effects [6]. So in this case, we gave the patient bortezomib weekly, but the appearance of mania was out of our imagine.

\section{Conclusions}

In summary, mania in a patient with $\mathrm{MM}$ is relatively rare. Prevention, timely diagnosis and clarifying the pathogenesis are the key steps for improvement. The common side effects of bortezomib are peripheral neuropathy, thrombocytopenia, neutropenia, ECG abnormalities, and seldom impaired liver function [7]. However, the alteration of mental state is rarely reported. It is urgently needed further preclinical studies focused on the mechanisms of MM and bortezomib on neuroendocrine system and direct rational drug use on MM patients.

\section{Acknowledgement}

None.

\section{Conflict of Interest}

All the authors declare no conflict of interest.

\section{References}

1. Lamers J, Hartmann M, Goldschmidt H, Brechtel A, Hillengass J, Herzog W. Psychosocial support in patients with multiple myeloma at time of diagnosis: who wants what? Psychooncology. 2013.

2. Song IS, Jeong YJ, Jeong SH, Heo HJ, Kim HK, Lee $\mathrm{SR}$, Ko TH, et al. Combination treatment with 2-methoxyestradiol overcomes bortezomib resistance of multiple myeloma cells. Exp Mol Med. 2013;45:e50.

3. Kelly K, Kalachand R, Murphy P. Bortezomib-induced reversible posterior leucoencephalopathy syndrome. Br J Haematol. 2008;141(5):566.

4. Terwiel E, Hanrahan R, Lueck C, D'Rozario J. Reversible posterior encephalopathy syndrome associated with bortezomib. Intern Med J. 2010;40(1):69-71.

5. Wojcik C, Di Napoli M. Ubiquitin-proteasome system and proteasome inhibition: new strategies in stroke therapy. Stroke. 2004;35(6):1506-1518.

6. Tokuhira M, Watanabe R, Nemoto T, Hanzawa K, Sagawa M, Tomikawa T, Mori S, et al. Successful treatment with a modified bortezomib schedule of weekly and longer intervals for patients with refractory/resistance multiple myeloma. Leuk Res. 2011;35(5):591-597.

7. Lu S, Wang J, Xu X, Ni X, Huang C, Qiu H, Hu X, et al. 
Bortezomib in combination with epirubicin, dexamethasone and thalidomide is a highly effective regimen in the treatment of multiple myeloma: a single-center experience. Int J Hematol. 2009;89(1):34-38. 